University of Wollongong

Research Online

Faculty of Engineering and Information

Faculty of Engineering and Information

Sciences - Papers: Part A

Sciences

$1-1-2015$

\title{
The role of pyrimidine and water as underlying molecular constituents for describing radiation damage in living tissue: A comparative study
}

Martina C. Fuss

Consejo Superior De Investigaciones Científicas

L Ellis-Gibbings

Consejo Superior de Investigaciones Cientificas

Darryl B. Jones

Flinders University

M J. Brunger

Flinders University, University of Malaya

Francisco Blanco

Universidad Complutense de Madrid

See next page for additional authors

Follow this and additional works at: https://ro.uow.edu.au/eispapers

Part of the Engineering Commons, and the Science and Technology Studies Commons

Research Online is the open access institutional repository for the University of Wollongong. For further information contact the UOW Library: research-pubs@uow.edu.au 


\title{
The role of pyrimidine and water as underlying molecular constituents for describing radiation damage in living tissue: A comparative study
}

\begin{abstract}
Water is often used as the medium for characterizing the effects of radiation on living tissue. However, in this study, charged-particle track simulations are employed to quantify the induced physicochemical and potential biological implications when a primary ionising particle with energy $10 \mathrm{keV}$ strikes a medium made up entirely of water or pyrimidine. Note that pyrimidine was chosen as the DNA/RNA bases cytosine, thymine, and uracil can be considered pyrimidine derivatives. This study aims to assess the influence of the choice of medium on the charged-particle transport, and identify how appropriate it is to use water as the default medium to describe the effects of ionising radiation on living tissue. Based on the respective electron interaction cross sections, we provide a model, which allows the study of radiation effects not only in terms of energy deposition (absorbed dose and stopping power) but also in terms of the number of induced molecular processes. Results of these parameters for water and pyrimidine are presented and compared.
\end{abstract}

\section{Keywords}

study, describing, comparative, constituents, molecular, underlying, water, pyrimidine, role, tissue, living, damage, radiation

\section{Disciplines}

Engineering | Science and Technology Studies

\section{Publication Details}

Fuss, M. C., Ellis-Gibbings, L., Jones, D. B., Brunger, M. J., Blanco, F., Muñoz, A., Limao-Vieira, P. \& García, G. (2015). The role of pyrimidine and water as underlying molecular constituents for describing radiation damage in living tissue: A comparative study. Journal of Applied Physics, 117 (21), 214701-1-214701-8.

\section{Authors}

Martina C. Fuss, L Ellis-Gibbings, Darryl B. Jones, M J. Brunger, Francisco Blanco, A Muñoz, Paulo LimaoVieira, and Gustavo Garcia 


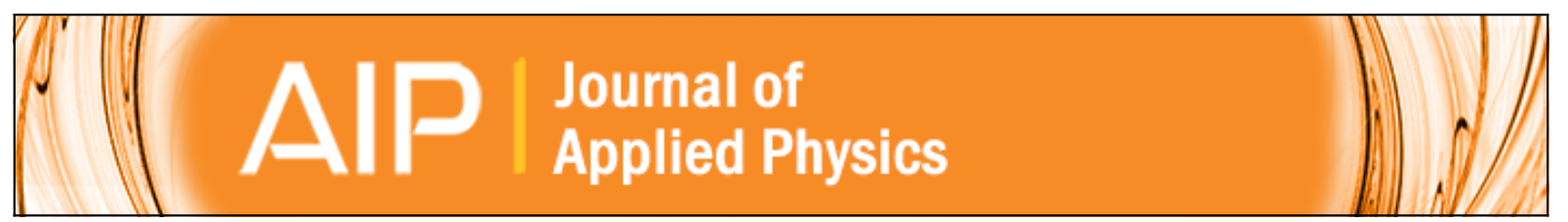

\section{The role of pyrimidine and water as underlying molecular constituents for describing radiation damage in living tissue: $A$ comparative study}

M. C. Fuss, L. Ellis-Gibbings, D. B. Jones, M. J. Brunger, F. Blanco, A. Muñoz, P. Limão-Vieira, and G. García

Citation: Journal of Applied Physics 117, 214701 (2015); doi: 10.1063/1.4921810

View online: http://dx.doi.org/10.1063/1.4921810

View Table of Contents: http://scitation.aip.org/content/aip/journal/jap/117/21?ver=pdfcov

Published by the AIP Publishing

\section{Articles you may be interested in}

Correlation between energy deposition and molecular damage from Auger electrons: A case study of ultra-low energy $(5-18 \mathrm{eV})$ electron interactions with DNA

Med. Phys. 41, 072502 (2014); 10.1118/1.4881329

Investigations of DNA damage induction and repair resulting from cellular exposure to high dose-rate pulsed proton beams

AIP Conf. Proc. 1546, 96 (2013); 10.1063/1.4816615

Influence of organic ions on DNA damage induced by $1 \mathrm{eV}$ to $60 \mathrm{keV}$ electrons

J. Chem. Phys. 133, 155102 (2010); 10.1063/1.3505046

Protection by organic ions against DNA damage induced by low energy electrons

J. Chem. Phys. 132, 045102 (2010); 10.1063/1.3298895

Radiation Protection Dosimetry: A Radical Reappraisal

Med. Phys. 26, 2047 (1999); 10.1118/1.598829

Frustrated by

old technology?

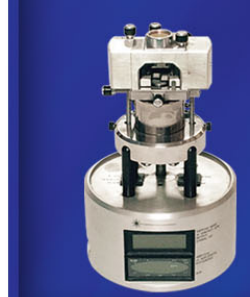

Is your AFM dead

and can't be repaired?

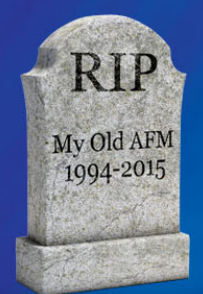

Sick of bad customer support?

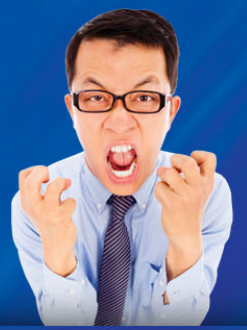

It is time to upgrade your AFM

Minimum $\$ 20,000$ trade-in discount for purchases before August 31st

Asylum Research is today's technology leader in AFM 


\title{
The role of pyrimidine and water as underlying molecular constituents for describing radiation damage in living tissue: A comparative study
}

\author{
M. C. Fuss, ${ }^{1}$ L. Ellis-Gibbings, ${ }^{1}$ D. B. Jones, ${ }^{2}$ M. J. Brunger, ${ }^{2,3}$ F. Blanco, ${ }^{4}$ A. Muñoz, ${ }^{5}$ \\ P. Limão-Vieira, ${ }^{2,6}$ and G. García ${ }^{1,7, a)}$ \\ ${ }^{1}$ Instituto de Física Fundamental, Consejo Superior de Investigaciones Científicas (CSIC), \\ Serrano 113-bis, 28006 Madrid, Spain \\ ${ }^{2}$ School of Chemical and Physical Sciences, Flinders University, GPO Box 2100, Adelaide, \\ South Australia 5001, Australia \\ ${ }^{3}$ Institute of Mathematical Sciences, University of Malaya, 50603 Kuala Lumpur, Malaysia \\ ${ }^{4}$ Departamento de Física Atómica, Molecular y Nuclear, Universidad Complutense de Madrid, \\ Avenida Complutense, 28040 Madrid, Spain \\ ${ }^{5}$ Centro de Investigaciones Energéticas Medioambientales y Tecnológicas, Avenida Complutense 22, \\ 28040 Madrid, Spain \\ ${ }^{6}$ Laboratório de Colisões Atómicas e Moleculares, CEFITEC, Departamento de Física, Faculdade \\ de Ciências e Tecnologia, Universidade Nova de Lisboa, 2829-516 Caparica, Portugal \\ ${ }^{7}$ Centre for Medical Radiation Physics, University of Wollongong, Wollongong, NSW 2522, Australia
}

(Received 6 February 2015; accepted 16 May 2015; published online 1 June 2015)

\begin{abstract}
Water is often used as the medium for characterizing the effects of radiation on living tissue. However, in this study, charged-particle track simulations are employed to quantify the induced physicochemical and potential biological implications when a primary ionising particle with energy $10 \mathrm{keV}$ strikes a medium made up entirely of water or pyrimidine. Note that pyrimidine was chosen as the DNA/RNA bases cytosine, thymine, and uracil can be considered pyrimidine derivatives. This study aims to assess the influence of the choice of medium on the charged-particle transport, and identify how appropriate it is to use water as the default medium to describe the effects of ionising radiation on living tissue. Based on the respective electron interaction cross sections, we provide a model, which allows the study of radiation effects not only in terms of energy deposition (absorbed dose and stopping power) but also in terms of the number of induced molecular processes. Results of these parameters for water and pyrimidine are presented and compared. (C) 2015 AIP Publishing LLC. [http://dx.doi.org/10.1063/1.4921810]
\end{abstract}

\section{INTRODUCTION}

Exposing living tissue to ionising radiation has become routine practice in clinical medicine for diagnostic purposes, and in particular for cancer therapeutic treatments. The biological effects of such radiation are known to be essentially produced by the secondary species generated along the radiation tracks and their subsequent reactions within the irradiated biological environment. ${ }^{1}$ These species can cause mutagenic, genotoxic, and other potentially lethal DNA lesions. ${ }^{2}$ Secondary electrons are the most abundant of the secondary species produced by the primary interaction, typically $5 \times 10^{4} / \mathrm{MeV}$ of incident radiation, ${ }^{3,4}$ and can efficiently induce damage at the molecular level, i.e., breaking chemical bonds that leads to molecular dissociations. ${ }^{1}$ This produces large quantities of highly reactive radicals, cations, and anions. The vast majority of these secondary electrons are created with energies typically below $30 \mathrm{eV} .{ }^{5,6}$ The ballistic electrons, before reaching thermalization, lose their kinetic energies through successive inelastic interactions (e.g., ionisation, excitation, dissociation, etc.) or can alternatively, attach to atoms and molecules in the medium, or hydrate in the medium itself to induce further

\footnotetext{
a) Author to whom correspondence should be addressed. Electronic mail: g.garcia@csic.es. Tel.: +34915616800 ext. 943214. Fax: +34 91 5854894 .
}

chemical changes. ${ }^{7}$ Greater control of radiation-induced chemical processes within the biological system will therefore facilitate desirable improvements in radiotherapeutic treatments, such as site-specific damage and minimizing damage to surrounding healthy tissue. This can only be achieved through a more detailed understanding of the processes occurring between the primary and secondary particles, and the molecules constituting biological systems. ${ }^{8}$

In order to quantitatively assess radiation dose and radiation-induced damage, there has been a concerted effort to develop Monte-Carlo simulations for describing chargedparticle interactions within living tissue. Some of the popular simulation codes include the Geometry and Tracking 4 (GEANT4), ${ }^{9,10}$ Penetration and Energy Loss of Positrons and Electrons (PENELOPE), ${ }^{11}$ Electron and Positron Transport (EPOTRAN), ${ }^{12}$ and the Low-Energy Particle Track Simulations (LEPTS). ${ }^{13}$ The inherent limitations in these current models are two-fold, as we now discuss below.

First, the simulations are largely carried out using gaseous or liquid water as a representation of the biological medium. ${ }^{14}$ Recently, the modelling of water in gas or liquid phases has been demonstrated to influence charged-particle transport. ${ }^{15}$ Here, the only difference between the cross sections (CSs) employed in the two models was a structure factor that "converted" gas-phase data into data suitable for the liquid phase. More recently, charged-particle transport in 
different $\mathrm{He}: \mathrm{H}_{2} \mathrm{O}^{16}$ and THF: $\mathrm{H}_{2} \mathrm{O}^{17}$ gas mixtures has been observed to produce a behaviour that is different from that of either of the pure gases. This highlights the sensitivity of charged-particle transport phenomena to the "make-up" of the medium. Here, we note that work has begun aiming to quantify radiation damage through prediction of single and double strand breaks in DNA through collaborative projects, like GEANT4-DNA. ${ }^{18}$ However, those simulations are still currently only available for liquid water.

The second limitation is that the relevant codes often have somewhat inadequate descriptions for how low-energy electrons interact with the constituent particles. In particular, some codes actually stop modelling secondary electrons if their energies drop below $50 \mathrm{eV} .{ }^{13}$ This may reflect a historical deficiency in the availability of reliable fundamental data, which can be incorporated into these models, but it nonetheless needs to be addressed. In particular, in recent years, substantial experimental and theoretical progress has been made, through a combined effort, to provide the essential data that describes how low-energy electrons interact with the key molecular building blocks of living tissue, i.e., water and molecular analogues to the structural components of DNA. In this paper, we carry out charged-particle track simulations in pyrimidine $\left(\mathrm{C}_{4} \mathrm{H}_{4} \mathrm{~N}_{2}\right)$ using the LEPTS code, and compare these results against those for water. Pyrimidine is a prototypical structure for the RNA/DNA bases thymine, cytosine, and uracil. This has made pyrimidine the subject of numerous experimental and theoretical investigations ${ }^{19-35}$ that provide a near complete characterization of pyrimidine's structure and scattering cross sections. These previous findings are presented here to form a nearcomplete database for pyrimidine, including theoretical and experimental cross section data for elastic and discrete inelastic scattering and ionization and attachment processes. As such, it is now possible to study pyrimidine in chargedparticle transport simulations. Pyrimidine, being a larger molecule that contains many more atoms than water, possesses many additional energy deposition channels than those available for water. Further, key molecular properties will also influence the charged-particle transport. Here, the LEPTS package describes low-energy electron interactions down to thermalisation (of the order of milli-electron-volts). Our pyrimidine results are compared to identical simulations performed when water is used as the transport medium. In this way, we can begin to assess the limitations and influence of selecting water as a default transport medium in simulations of radiation damage, and perhaps choose other background species or mixture of species that are more attuned to represent generic molecular systems.

In Sec. II, we present the details on our LEPTS programme, whereas in Sec. III we briefly describe the methodology and data sources. This is followed by the presentation and discussion of our results in Sec. IV. Finally, in Sec. V, the conclusions from this study are drawn.

\section{LEPTS PROGRAMME}

The LEPTS code has been described in detail elsewhere $^{36}$ and so only a brief description is given here. Briefly, the programme is based on critically selected experimental and theoretical input data previously compiled for each target molecule, in this particular case for water and pyrimidine. The LEPTS physics model is designed to give a comprehensive description of the underlying mechanisms of electron (and positron) interactions with the relevant target molecules, i.e., it provides detailed information on each particle-molecule interaction event. Such interactions are typically described in an energy region below $10 \mathrm{keV}$, where standard approximations such as the Born-Bethe theory become unsuitable, down to thermal energies. ${ }^{37}$ Therefore, the Monte Carlo code LEPTS ${ }^{38}$ is an event-by-event simulation procedure that runs in a $\mathrm{C}^{++}$environment, which is compatible, and can be combined, with other general purpose Monte Carlo codes like GEANT4 ${ }^{8}$ and PENELOPE. ${ }^{10}$ As such, it can be used for expanding the energy range upwards, or for simulating other types of primary radiation (such as happens with photons). Other related tools, like the GEANT4 code, are used to define the target materials and geometries. While tracking an incident particle throughout the energy deposition process, elastic collisions and different types of inelastic interactions are distinguished and modelled according to the underlying input data. As such, for each collisional event, the programme samples the scattering angle and the particle's energy loss specifically according to the corresponding distribution functions, ${ }^{36}$ which are the differential cross section (DCS) data and the energy loss spectra. The programme is also capable of providing an efficient response if an ionisation event takes place. A secondary electron is immediately generated and will enter the simulation process with its energy and direction given by applying energy conservation and linear momentum conservation. All the particles, including the secondary ones, are followed until thermalisation is reached. After the simulation is completed, 3D maps of all the collisions produced in the course of the particles' thermalisation, together with information about the corresponding incident energy (immediately before the collision), energy deposition, particle type, and type of interaction, are provided with the possibility to obtain sufficient detail in particular, nanovolumes so as to investigate the nanodosimetry response.

\section{METHODOLOGY AND DATA SOURCES}

We have followed a similar methodology ${ }^{13}$ to that employed in our previous studies on other molecular targets, when modelling single electron tracks in biologically relevant media in order to assess molecular-level dosimetry. The information required to perform those simulations includes a considerable amount of scattering data in regards to integral and differential cross sections, and energy loss distribution functions. Briefly, these establish a self-consistent and complete set of interaction data and are compiled from both new and existing experimental and/or theoretical data for electron impact energies in the range of $1-10000 \mathrm{eV}$. For a comprehensive description, see Ref. 36 and references therein. When comparing the different studies published on electron scattering from pyrimidine, with regard to the compilation of a scattering data base, the most significant data sources are the 
vibrational and electronic excitation measurements on condensed pyrimidine by Levesque et al., ${ }^{31}$ the electronic-state integral cross sections and differential cross sections in Jones and colleagues, ${ }^{20-22}$ the differential elastic scattering experiments of Maljkovic et al. ${ }^{28}$ and Palihawadana et al. ${ }^{25}$ and the theoretical independent atom model with screening corrected additivity rule (IAM-SCAR) results for total scattering ${ }^{27}$ and integral elastic, inelastic, and rotational excitation. ${ }^{39}$ Other studies include the Schwinger multichannel (SMC) approach on elastic differential scattering as published in Palihawadana et al. ${ }^{25}$ and the R-matrix theory predictions of elastic scattering and electronic excitation, as well as the experimental differential electronic excitation CSs given in Masin et al..$^{22}$ Regarding ionisation, such measurements have been reported by Linert et $a l^{40}$ and, most recently, we note the total CS measurements by Baek et al. ${ }^{41}$ and by Fuss et al. ${ }^{26}$ Finally, we note the calculation of Ferraz et al., ${ }^{42}$ based on the scaled quasi-free-scattering model (SQFSM), and the experimental ionization results of Wolff et al. ${ }^{43}$ The cross sections are collated to give the recommended set plotted in Fig. 1, while a sample of our IAM-SCAR elastic DCS is given in Fig. 2. Input data and cross sections are summarised in Tables I and II, respectively, with a clarification regarding the nature of the original data (experimental, theoretical, or combination) they are based on.
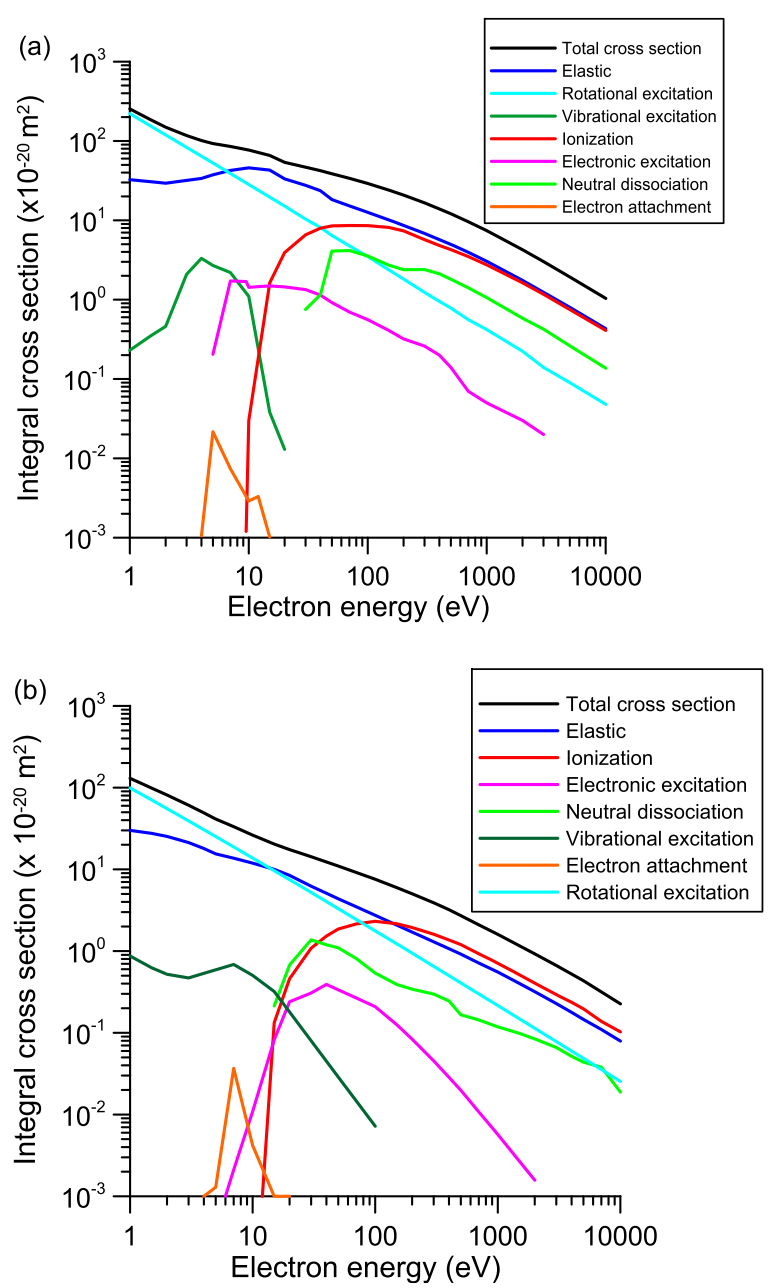

FIG. 1. Integral electron scattering cross sections for (a) pyrimidine (see text for details) and (b) water ${ }^{36}$ used for the simulations.

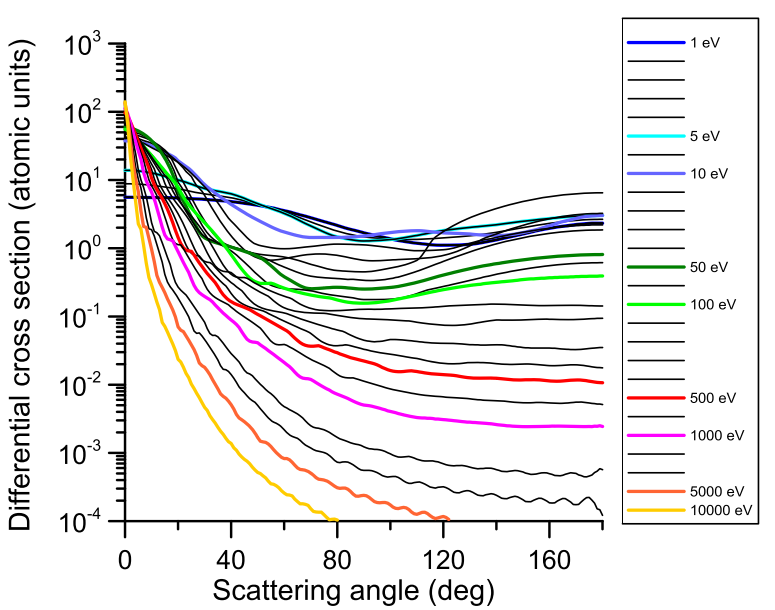

FIG. 2. Elastic differential cross sections used for the LEPTS simulation of electron interactions with pyrimidine.

\section{RESULTS AND DISCUSSION}

The database we employed for our water simulations has been described previously, ${ }^{36,37,44}$ and so we do not repeat that detail again here. Nonetheless, for completeness, the

TABLE I. Overview of the input parameters available for simulation with LEPTS. The origin of each dataset is indicated as experimental ("exp"), theoretical ("th"), approximation or derivation by other principles ("approx"), or a combination of any of these. The datasets listed cover at least the energy range of $1-10 \mathrm{keV}$.

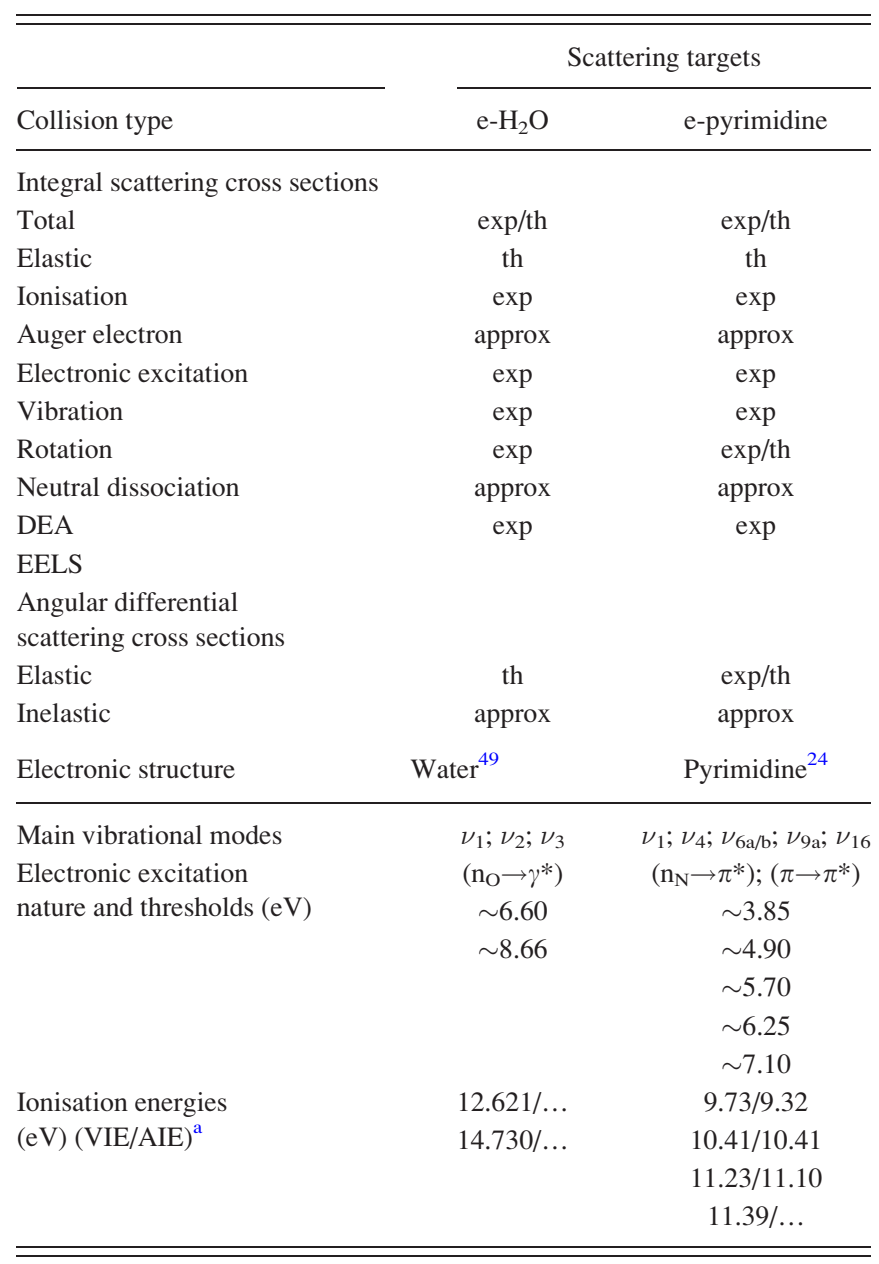

${ }^{a}$ VIE_vertical ionisation energy; AIE—adiabatic ionisation energy. 
relevant cross sections are also reproduced in Fig. 1(b). The LEPTS procedure for a given particle-target arrangement requires a proper database, which the present authors mainly prefer to use from available experimental sources rather than the calculations. Nonetheless, data from theoretical work are used if no conclusive experimental results are available. Here, the methodology used to select the most reliable studies is to compare data we have generated against all other available work, with the ultimate recommended set then being chosen. Another procedure pertains to results from distinct groups in different energy ranges. Here, we follow, as smoothly as possible, transitions from one source to another by taking average values, when necessary, of two data points in the overlapping region. However, if no data are available for an energy range and scattering process, in particular, in the high-energy domain (as it happens for most cases in the $\mathrm{keV}$ range), an interpolation is carried out using the closest recommended data points.

The first and initially most relevant set of data is the total CS, which equals the sum of all the other integral CSs, and serves therefore for checking the level of selfconsistence of the various integral CSs. For the majority of cases, the total cross section is the most accurate (typically within $5 \%-10 \%$ ) parameter due to the availability of several experimental and/or theoretical studies. Integral CSs for individual interaction processes are then examined roughly in the order of importance (i.e., in terms of the relative magnitude of the CS). The final recommended CS set for all the scattering processes is required to sum up exactly to the total $\mathrm{CS}$, for the entire energy range, in order to form an adequate input database for the track structure simulation. Fig. 1(a) gives an overview of the available and different pyrimidine total, elastic, and inelastic integral cross sections. In the intermediate energy range, the experimental CS values typically show good agreement with each other and with the IAM-SCAR theory and the sum of the different partial integral cross sections is well compatible with the total CS. For high energies, a less significant number of data are available but the total cross sections from all sources ${ }^{26,39,41}$ do nicely agree. In addition, the highest-energy experimental points on ionisation $^{43}$ are very compatible with the IAM-SCAR electronically inelastic $\mathrm{CSs}^{26}{ }^{26}$ However, the picture drawn from the total ${ }^{22,39,41,42}$ and integral elastic $\mathrm{CSs}^{22,25}$ from different sources is rather inhomogeneous in the lower energy range for both theory and the experiments. It should be noted that due to the considerable permanent dipole moment of pyrimidine ( 2.33 D (Ref. 45)), rotational excitation processes, causing scattering of the electrons into forward directions, gain a large weight compared to the other collisional interactions, particularly for the much lower incident energies. Unfortunately, experiments for determining total cross sections do not attain a sufficient energy resolution for discriminating against the small energy losses caused by rotational excitations, nor do they reach a geometrical acceptance angle low enough to effectively reject extremely forward-scattered electrons, introducing therefore systematic uncertainties in the measured values. Elastic CS measurements can in turn include some contributions from rotational excitations due to the energy resolution the spectrometer can attain. On the other hand, theoretical methods address this issue with different dipole "corrections" in order to obtain numerically convergent CS values, sometimes even in the case of elastic scattering. Summarizing, the observed discrepancies encountered between total and elastic cross section data from different sources at lower incident energies are closely related to the difficulties in accurately determining the contribution of rotational excitations in electron scattering from pyrimidine.

In order to obtain an accurate and self-consistent set of input data for the electron-pyrimidine interaction simulation, the most reliable partial cross sections (for the different processes) were first identified as the primary reference. In the case of the integral elastic CS, the purely elastic (non-dipolecorrected) CS obtained with a SMC method by Paliwahadana et al. $^{25}$ was used in the energy range $<50 \mathrm{eV}$ and completed with the IAM-SCAR results of Zecca et al. ${ }^{27}$ for energies $\geq 50 \mathrm{eV}$. The rotational excitation cross section was included as calculated in Ref. 39 for a free electric dipole. Vibrational excitations are taken from the values found by Levesque et $a l .{ }^{31}$ in the solid state. The integral CS values for electronic excitation utilized in the simulation are those derived by Jones et al., ${ }^{20}$ based on their differential measurements, and their extrapolation to higher incident energies. Electron-impact ionisation is incorporated using the experimental values of Linert et al. ${ }^{40}$ from threshold up to $100 \mathrm{eV}$, and the results of Wolff et al. ${ }^{43}$ for energies $\geq 150 \mathrm{eV}$. For still higher energies, data were extrapolated with the help of semi-experimental results gained from energy loss spectra measured in the Madrid laboratory. ${ }^{46}$ Finally, experimental integral cross sections for dissociative electron attachment (DEA) were supplied by Field. ${ }^{47}$

In the energy range noticeably affected by the dipoleinduced enhancement of rotational scattering $(0-20 \mathrm{eV})$, the total scattering CS was then obtained by summing up the aforementioned partial CSs. The resulting values are considerably higher than the experimental $\mathrm{data}^{26,41}$ and the SQFSM theory, ${ }^{42}$ but come to lie below the R-matrix and IAM-SCAR predictions which include dipole-induced rotations. ${ }^{26}$ Around $30 \mathrm{eV}$, the sum of the partial CSs comes into very good agreement with the IAM-SCAR calculation, so that the latter is used for energies $\geq 30 \mathrm{eV}$. The small differences between the total CS and the sum of partial CSs arising for energies $\geq 30 \mathrm{eV}$ are ascribed to neutral dissociation. A compilation of all the resulting integral cross sections is presented in Table II.

Regarding the elastic DCSs, we observe a situation very similar to the one found for tetrahydrofuran. ${ }^{48}$ The experimental angular distributions ${ }^{23,28}$ for low energies are very well reproduced by the un-Born-corrected SMC results ${ }^{25}$ (not shown), although some particular features in the intermediate angular range become less apparent with increasing energy. As the input for the simulation database, angular distributions are therefore derived mainly on the basis of the experimental data from Palihawadana et $a l^{25}$ and Maljkovic et $a l .{ }^{28}$ The theoretical distributions are used as a guide to extrapolate data towards the near-zero and extreme backward angles not covered by the experiments. For higher incident energies, the experimental DCSs reported by Maljkovic et $a l^{28}$ agree very well with the IAM-SCAR calculation; however, the calculated values offer the advantage of 
TABLE II. Integral cross sections $\left(\AA^{2}\right)$ selected for our LEPTS simulation for electron scattering from pyrimidine.

\begin{tabular}{|c|c|c|c|c|c|c|c|c|}
\hline Energy $(\mathrm{eV})$ & Total & Elastic & Rotation & Vibration & Electronic excitation & Ionisation & Neutral dissociation & Dissociative electron attachment \\
\hline 1.0 & 253.9 & 32.48 & 221.2 & 0.23 & $\ldots$ & $\ldots$ & $\ldots$ & 0.000192 \\
\hline 1.5 & 185.1 & 30.69 & 154.0 & 0.35 & $\ldots$ & $\ldots$ & $\ldots$ & 0.000168 \\
\hline 2.0 & 149.4 & 29.41 & 119.6 & 0.46 & $\ldots$ & $\ldots$ & $\ldots$ & 0.000144 \\
\hline 3.0 & 117.1 & 31.84 & 83.17 & 2.09 & $\ldots$ & $\ldots$ & $\ldots$ & 0.000288 \\
\hline 4.0 & 101.5 & 33.76 & 64.41 & 3.32 & $\ldots$ & $\ldots$ & $\ldots$ & 0.00106 \\
\hline 5.0 & 93.0 & 37.47 & 52.65 & 2.7 & 0.205 & $\ldots$ & $\ldots$ & 0.02161 \\
\hline 7.0 & 85.4 & 42.58 & 38.92 & 2.20 & 1.72 & $\ldots$ & $\ldots$ & 0.00738 \\
\hline 10 & 76.9 & 45.78 & 28.28 & 1.10 & 1.69 & 0.03 & $\ldots$ & 0.00286 \\
\hline 15 & 65.7 & 42.97 & 19.60 & 0.038 & 1.43 & 1.63 & $\ldots$ & $\ldots$ \\
\hline 20 & 53.8 & 33.25 & 15.12 & 0.013 & 1.46 & 3.93 & $\ldots$ & $\ldots$ \\
\hline 30 & 46.8 & 27.6 & 10.36 & $\ldots$ & 1.49 & 6.56 & 0.754 & $\ldots$ \\
\hline 40 & 42.3 & 23.6 & 8.121 & $\ldots$ & 1.45 & 7.96 & 1.150 & $\ldots$ \\
\hline 50 & 38.6 & 18.3 & 6.441 & $\ldots$ & 1.34 & 8.51 & 4.091 & $\ldots$ \\
\hline 70 & 33.9 & 15.2 & 4.760 & $\ldots$ & 1.14 & 8.61 & 4.163 & $\ldots$ \\
\hline 100 & 29.1 & 12.6 & 3.500 & $\ldots$ & 0.92 & 8.57 & 3.555 & $\ldots$ \\
\hline 150 & 24.1 & 10.11 & 2.380 & $\ldots$ & 0.70 & 8.14 & 2.727 & $\ldots$ \\
\hline 200 & 20.8 & 8.65 & 1.820 & $\ldots$ & 0.56 & 7.36 & 2.387 & $\ldots$ \\
\hline 300 & 16.6 & 6.83 & 1.232 & $\ldots$ & 0.41 & 5.71 & 2.392 & $\ldots$ \\
\hline 400 & 13.9 & 5.74 & 0.952 & $\ldots$ & 0.32 & 4.82 & 2.116 & $\ldots$ \\
\hline 500 & 12.1 & 4.96 & 0.784 & $\ldots$ & 0.26 & 4.28 & 1.811 & $\ldots$ \\
\hline 700 & 9.60 & 3.95 & 0.560 & $\ldots$ & 0.20 & 3.49 & 1.408 & $\ldots$ \\
\hline 1000 & 7.42 & 3.05 & 0.420 & $\ldots$ & 0.14 & 2.75 & 1.062 & $\ldots$ \\
\hline 2000 & 4.26 & 1.75 & 0.224 & $\ldots$ & 0.07 & 1.63 & 0.584 & $\ldots$ \\
\hline 3000 & 3.02 & 1.24 & 0.140 & $\ldots$ & 0.05 & 1.17 & 0.426 & $\ldots$ \\
\hline 5000 & 1.93 & 0.80 & 0.090 & $\ldots$ & 0.03 & 0.75 & 0.262 & $\ldots$ \\
\hline 10000 & 1.04 & 0.431 & 0.048 & $\ldots$ & 0.02 & 0.41 & 0.137 & $\ldots$ \\
\hline
\end{tabular}

covering the complete angular range on a $1^{\circ}$ grid, as is necessary for the simulation input. These theoretical results are thus used for incident energies $\geq 70 \mathrm{eV}$. The resulting angular distributions for some of the energies included in the database are presented in Fig. 2.

In addition to integral and differential scattering cross sections, energy loss distributions are also required for an accurate simulation of the energy deposition caused in a molecular material. Experimental electron energy loss spectra for pyrimidine have been presented by Colmenares et al. ${ }^{46}$ for a range of incident energies (30-2000 eV). Examining these spectra, one notes a significant increase in the average energy loss as the incident energy increases. This affects the electronic excitation region of the spectra, where the main distinguishing discrete inelastic peak is more prominent for lower incident energies, and the part of the spectrum near and after the ionization maximum, which is shifted towards higher energy losses and presents a slower decay for the higher incident energies. Consequently, for the simulation we use two representative electron energy loss distributions that are based on the low-energy and high-energy measurements, depending on the incident electron's energy $(\leq 50 \mathrm{eV}$ or $>50 \mathrm{eV}$ ). The average energy loss of a charged particle per unit path length, when passing through an absorber, together with the cross section for inelastic scattering, can now be used to derive the stopping power. We assume that the total stopping power $\mathrm{S}$ equals the collisional stopping power $\mathrm{S}_{\mathrm{col}}$, since radiative energy losses are negligible in the energy range studied in the present work. For a detailed description and justification, see Ref. 46. Since here we are aiming at a comparative study of the behaviour in pyrimidine against that in water, Fig. 3 shows the stopping power data for both molecular targets. Note that in the case of pyrimidine we are extending the energy range being studied up to $10000 \mathrm{eV}$, a higher energy than that considered in our preliminary work. ${ }^{46}$ For both targets, the overall uncertainty on the results in Fig. 3 amounts to about $20 \%(\mathrm{E} \leq 50 \mathrm{eV})$ or $15 \%$ $(\mathrm{E}>300 \mathrm{eV})$, when taking into account the uncertainties associated with the inelastic CSs and the mean energy losses. Although the trend of both curves seems to qualitatively converge at higher energies $(>100 \mathrm{eV})$, with mutual differences in magnitude of $\sim 16 \%$, in the low energy range $(<20 \mathrm{eV})$

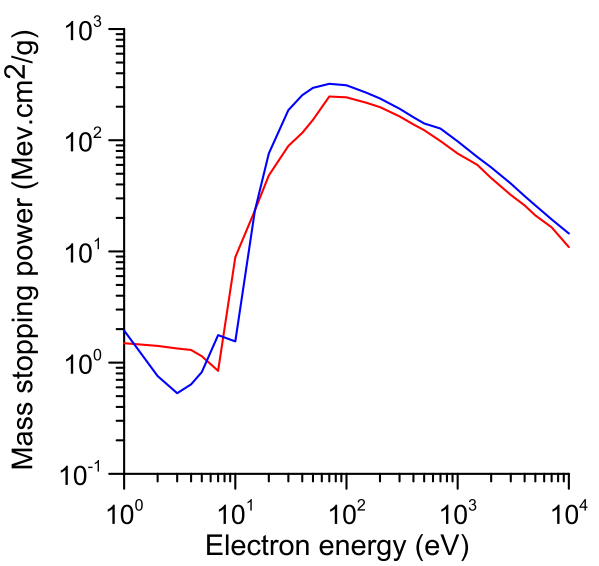

FIG. 3. Comparison of the pyrimidine (red line) and water (blue line) mass stopping powers as a function of the electron impact energy. Note the $\mathrm{x}$ - and $\mathrm{y}-\log$ scales. See text for further details. 
the differences are more noticeable and are certainly due to the different CSs of both species at lower energies (see Fig. 1) and to the role of the molecular structure where the excitation/ionisation thresholds differ from pyrimidine ${ }^{23}$ to water ${ }^{49}$ (see Table I bottom). Note that the changes of slope in the stopping power curves for water and pyrimidine shown in Fig. 3 are due to changes in the corresponding cross section values (due to resonances or to the opening of inelastic channels).

As mentioned earlier, cross section data and energy loss distribution functions for both water and pyrimidine have been used in the LEPTS code to simulate single electron tracks. ${ }^{36}$ As indicated in Ref. 36, we use, as input parameters, data for single molecules (in the gas phase) but electron tracks are modelled in the liquid phase by considering the liquid density and correcting the cross section values in order to introduce screening effects from the surrounding molecules (see Ref. 36 for details). In order to show their characteristics, in particular, for possible applications and differences of this modelling procedure, for electrons in pyrimidine and water with initial energies of $10 \mathrm{keV}$, the number of interactions as a function of the depth $(\mu \mathrm{m})$ for each scattering process or group of common processes (all inelastic excitations) are shown in Figs. 4 and 5, respectively. Under these conditions, single tracks for $10^{5}$ electrons, until their final thermalisation, have been included (see Fig. 6), where we reiterate that each characteristic scattering channel in Figs. 4 and 5 represents the total sum of that particular process. Considering Figs. 4 and 5 in more detail, we notice that the maximum penetration of $10 \mathrm{keV}$ electrons in water is around $1.5 \mu \mathrm{m}$, whereas in the case of pyrimidine it reaches $1.9 \mu \mathrm{m}$. Moreover, rotational excitation processes are more relevant in pyrimidine than with respect to water (where elastic scattering dominates), which can be related to higher dipole moment of the former $(2.33 \mathrm{D})$ in contrast to the latter $(1.85 \mathrm{D}){ }^{45}$ The track structures (see Fig. 6), resulting from the underlying processes, and leading to the results presented in Figs. 4 and 5, allow therefore a detailed evaluation of the energy deposition as a function of the electron depth in the medium, which is shown in Fig. 7. It is clear that there are differences observed in the energy deposition profiles between pyrimidine and water, although both show the same

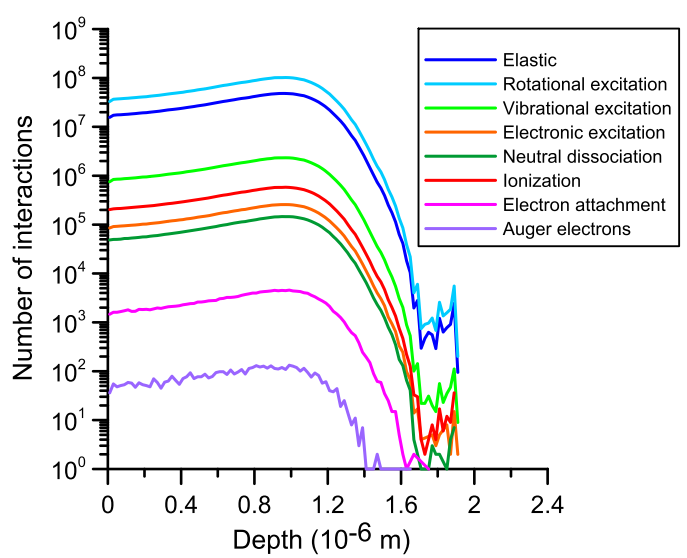

FIG. 4. Number of interactions as a function of the depth $(\mu \mathrm{m})$ for electron induced processes in pyrimidine. See text for further details.

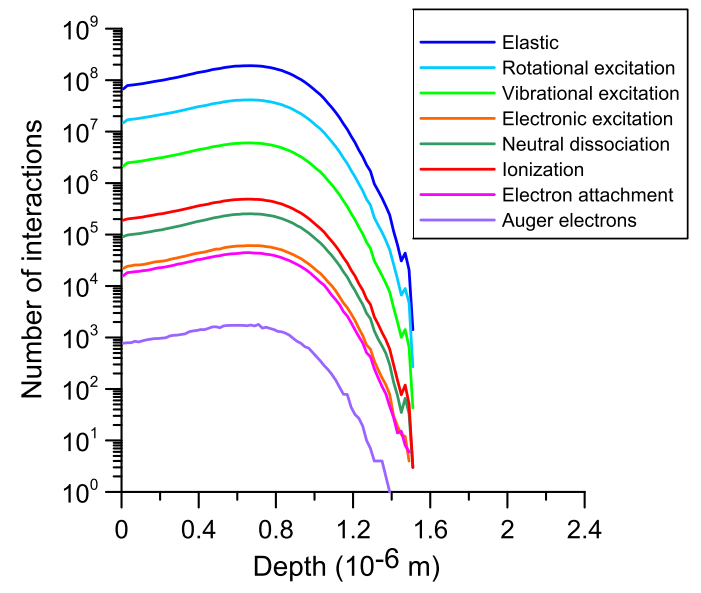

FIG. 5. Number of interactions as a function of the depth $(\mu \mathrm{m})$ for electron induced processes in water. See text for further details.

monotonically decreasing trend after the maxima. In the lower depth region (before the maximum), the energy deposition is enhanced in the case of water with a maximum value higher than that found in pyrimidine. However, the range of penetration in the two media is favoured in the case of pyrimidine. This result is not particularly surprising, and is consistent with the nature of the lowest-lying excited states that can be attained for lower energies in pyrimidine compared to water, i.e., the electronic structure is certainly relevant here as the electrons are being slowed down by inelastic processes before thermalisation and the nature of the probabilities (cross sections) for those various processes. Considering now the information we obtained in Fig. 6, we find a 3D map for the whole irradiated volume as well as for selected nanovolumes close to the end of the electron track. We anticipate that for the latter region, the energy deposition may lead to a meaningless absorbed dose for it. Fig. 6(a) represents a typical electron track, where energy loss processes occurring in single collisions and the type of process that is

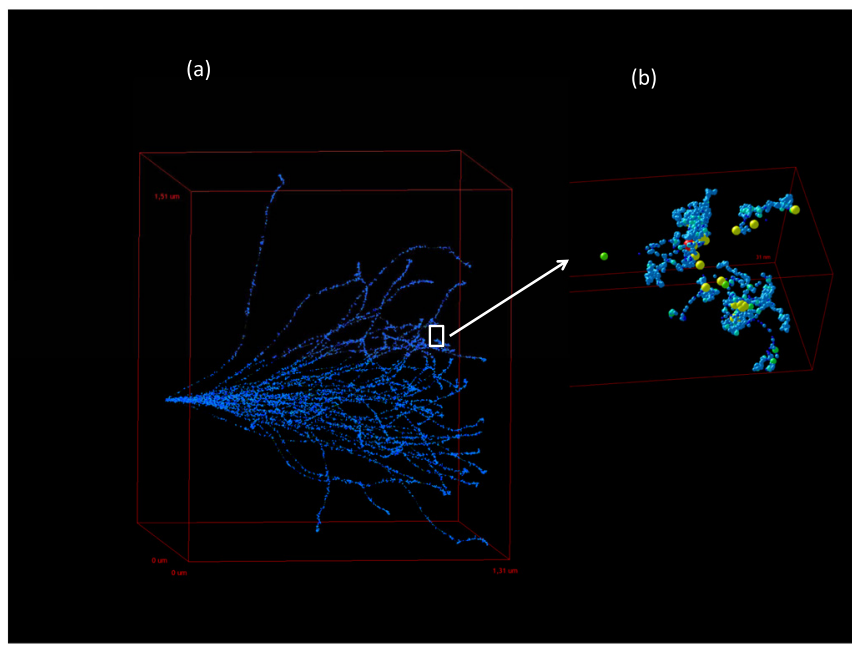

FIG. 6. Charged-particle track simulations in liquid pyrimidine as a function of depth $(\mu \mathrm{m})$. (a) $10^{5}$ electrons with $5 \mathrm{keV}$ incident energy slowing down by successive collisions (coloured balls). (b) Nanovolume detail close to the end of a selected track. Type of interactions: $\bullet$, elastic scattering; $\bullet$, rotational excitation; $\bullet$, vibrational excitation; $\bullet$, electronic excitation; $\bullet$, neutral dissociation; $\bullet$, ionisation. 


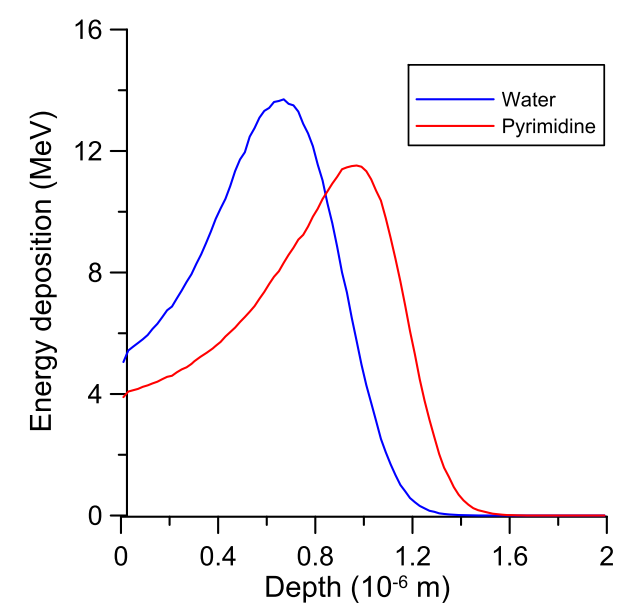

FIG. 7. Energy deposition of $10^{5}$ incident electrons each with $10 \mathrm{keV}$ energy in liquid water and pyrimidine (1.000 and $1.016 \mathrm{~g} / \mathrm{cm}^{3}$ density, respectively), as a function of depth $(\mu \mathrm{m})$.

taking place, respectively, are represented. For the chargedparticle track simulations presented here, electrons are released into liquid pyrimidine with an initial energy of $5 \mathrm{keV}$ and are then tracked until thermalisation occurs. While the corresponding water results, under identical initial conditions, are not shown here, we note that the determined track structure is very different than that seen for pyrimidine in Fig. 6(a). This observation, at least in part, reflects the very different cross sections for both species (see Fig. 1). In Fig. 6(b), the final portion of a single track ending can be seen. Due to the molecular-level description of all collisional events that is achieved by the LEPTS programme, a wide range of detailed information on the underlying processes, such as the type of interaction and exact energy loss at each collision point, becomes available in addition to the more common transport information (not shown here). Of particular relevance is the presence of secondary electrons being produced as additional tracks emerging from the main trajectory, which are clearly identified from the large density of tracks pertaining to the elastic scattering process. Notwithstanding this point, several tens of dissociative processes in the volume are also seen to be inducing damage in regard to bond breaking and structural modifications of the medium molecular constituents. In such a description, we define nano-dosimetry as the result of dissociation induced damage at the molecular level rather than the absorbed dose. Therefore, the simulation procedure implemented in this methodology can be considered as a useful nano-dosimetric tool for applications requiring such a detailed level of description, i.e., at the molecular level. Whether pyrimidine can actually be considered as a better mimic of a physiological environment, to the detriment of water, or whether both and yet further species are needed for a quantitative representation of such environment, still requires further extensive studies with particular relevance for experimental data. As far as the authors' are aware, no previous results for pyrimidine from simulation codes in the energy range of $1-10000 \mathrm{eV}$, as considered here, have been reported in the literature. We believe this reflects a hitherto lack of proper experimental data being available.

\section{CONCLUSION}

We have provided a complete set of differential and integral electron scattering cross section data for pyrimidine, based on our previous experiments and together with calculations and other data available in the literature. Quantitative and detailed knowledge of the underlying processes (integral and differential cross sections and energy loss spectra) resulting from the interaction of electrons $(1-10000 \mathrm{eV})$ with pyrimidine, and water molecules, have been used as input parameters to describe the interaction model in the LEPTS code. Electrons as incoming particles were found to have a higher penetration depth for pyrimidine $(1.9 \mu \mathrm{m})$ than for water $(1.5 \mu \mathrm{m})$. This may have considerable biological consequences within the physiological environment regarding how effective dose is evaluated within nanovolumes in targeted radiation therapies. It certainly indicates that the behaviour of electrons traversing through pyrimidine is different than when they traverse through water. Therefore, the present results strongly suggest that using water as the default medium for biological systems might not be quantitatively correct. We believe that the two main reasons which explain our different observations for electron tracks in pyrimidine vis a vis water relate to both their very different electronic structures and their different electron scattering and interaction probabilities (i.e., the cross sections). The present results therefore provide a transparent example for how the behaviour at the nanoscale drives the macroscopic behaviour of the system, which is described by terms such as the stopping power or energy deposition. Using a complete set of realistic cross section data, we have shown that radiation effects can be described not only in terms of energy deposition but also in terms of induced molecular processes. At this stage, the present modeling procedure provides information on the number and type of molecular dissociations induced by low energy electrons but additional information on further interactions of the created radicals with the constituent of the medium would be desirable and will be the subject of future investigations.

\section{ACKNOWLEDGMENTS}

This research was supported by the Australian Research Council (ARC) through its Centres of Excellence Program. D.B.J. thanks the ARC for provision of a Discovery Early Career Researcher Award. We also acknowledge the support of the Spanish Ministerio de Economia y Competitivad under Project No. FIS 2012-31230 and the European Union COST Actions (MP1002 and CM1301). P.L.V. acknowledges the Portuguese Foundation for Science and Technology (FCTMEC) through research grants PTDC/FIS-ATO/1832/2012, UID/FIS/00068/2013, and SFRH/BSAB/105792/2014. P.L.V. also acknowledges his Visiting Professor position at Flinders University, Adelaide, South Australia.

\footnotetext{
${ }^{1}$ B. Boudaiffa, P. Cloutier, D. Hunting, M. A. Huels, and L. Sanche, Science 287, 1658 (2000).

${ }^{2}$ C. von Sonntag, Free-Radical-Induced DNA Damage and its Repair (Springer, New York, 2005).
} 
${ }^{3}$ L. Sanche, in Radiation Induced Molecular Phenomena in Nucleic Acids, edited by M. Shukla and J. Leszczynski (Springer, Netherlands, 2008), Vol. 5, pp. 531-575.

${ }^{4}$ S. M. Pimblott and J. A. LaVerne, Radiat. Phys. Chem. 76, 1244 (2007).

${ }^{5}$ V. Cobut, Y. Frongillo, J. P. Patau, T. Goulet, M.-J. Fraser, and J.-P. JayGerin, Radiat. Phys. Chem. 51, 229 (1998).

${ }^{6}$ J. A. LaVerne and S. Pimblott, Radiat. Res. 141, 208 (1995).

${ }^{7}$ L. Turi and P. J. Rossky, Chem. Rev. 112, 5641-5674 (2012).

${ }^{8}$ L. Sanche, Nature 461, 358-359 (2009).

${ }^{9}$ S. Agostinelli, J. Allison, K. Amako, J. Apostolakis, H. Araujo, P. Arce, M. Asai, D. Axen, S. Banerjee, G. Barrand, F. Behner, L. Bellagamba, J. Boudreau, L. Broglia, A. Brunengo, H. Burkhardt, S. Chauvie, J. Chuma, R. Chytracek, G. Cooperman, G. Cosmo, P. Degtyarenko, A. Dell'Acqua, G. Depaola, D. Dietrich, R. Enami, A. Feliciello, C. Ferguson, H. Fesefeldt, G. Folger, F. Foppiano, A. Forti, S. Garelli, S. Giani, R. Giannitrapani, D. Gibin, J. J. Gómez Cadenas, I. González, G. Gracia Abril, G. Greeniaus, W. Greiner, V. Grichine, A. Grossheim, S. Guatelli, P. Gumplinger, R. Hamatsu, K. Hashimoto, H. Hasui, A. Heikkinen, A. Howard, V. Ivanchenko, A. Johnson, F. W. Jones, J. Kallenbach, N. Kanaya, M. Kawabata, Y. Kawabata, M. Kawaguti, S. Kelner, P. Kent, A. Kimura, T. Kodama, R. Kokoulin, M. Kossov, H. Kurashige, E. Lamanna, T. Lampén, V. Lara, V. Lefebure, F. Lei, M. Liendl, W. Lockman, F. Longo, S. Magni, M. Maire, E. Medernach, K. Minamimoto, P. Mora de Freitas, Y. Morita, K. Murakami, M. Nagamatu, R. Nartallo, P. Nieminen, T. Nishimura, K. Ohtsubo, M. Okamura, S. O'Neale, Y. Oohata, K. Paech, J. Perl, A. Pfeiffer, M. G. Pia, F. Ranjard, A. Rybin, S. Sadilov, E. Di Salvo, G. Santin, T. Sasaki, N. Savvas, Y. Sawada, S. Scherer, S. Sei, V. Sirotenko, D. Smith, N. Starkov, H. Stoecker, J. Sulkimo, M. Takahata, S. Tanaka, E. Tcherniaev, E. Safai Tehrani, M. Tropeano, P. Truscott, H. Uno, L. Urban, P. Urban, M. Verderi, A. Walkden, W. Wander, H. Weber, J. P. Wellisch, T. Wenaus, D. C. Williams, D. Wright, T. Yamada, H. Yoshida, and D. Zschiesche, Nucl. Instrum. Methods Phys. Res., Sect. A 506, 250-303 (2003).

${ }^{10}$ J. Allison, K. Amako, J. Apostolakis, H. Araujo, P. A. Dubois, M. Asai, G. Barrand, R. Capra, S. Chauvie, R. Chytracek, G. A. P. Cirrone, G. Cooperman, G. Cosmo, G. Cuttone, G. G. Daquino, M. Donszelmann, M. Dressel, G. Folger, F. Foppiano, J. Generowicz, V. Grichine, S. Guatelli, P. Gumplinger, A. Heikkinen, I. Hrivnacova, A. Howard, S. Incerti, V. Ivanchenko, T. Johnson, F. Jones, T. Koi, R. Kokoulin, M. Kossov, H. Kurashige, V. Lara, S. Larsson, F. Lei, O. Link, F. Longo, M. Maire, A. Mantero, B. Mascialino, I. McLaren, P. M. Lorenzo, K. Minamimoto, K. Murakami, P. Nieminen, L. Pandola, S. Parlati, L. Peralta, J. Perl, A. Pfeiffer, M. G. Pia, A. Ribon, P. Rodrigues, G. Russo, S. Sadilov, G. Santin, T. Sasaki, D. Smith, N. Starkov, S. Tanaka, E. Tcherniaev, B. Tome, A. Trindade, P. Truscott, L. Urban, M. Verderi, A. Walkden, J. P. Wellisch, D. C. Williams, D. Wright, and H. Yoshida, IEEE Trans. Nucl. Sci. 53, 270-278 (2006).

${ }^{11}$ F. Salvat, J. M. Fernandez-Varea, and J. Sempau, in PENELOPE2011: A Code System for Monte-Carlo Simulation of Electron and Photon Transport, OECD—Nuclear Energy Agency, 2011.

${ }^{12}$ C. Champion, C. Le Loirec, and B. Stosic, Int. J. Radiat. Biol. 88, 54-61 (2012).

${ }^{13}$ A. Muñoz, F. Blanco, J. C. Oller, J. M. Pérez, and G. García, in Advances in Quantum Chemistry, edited by J. R. Sabin and E. Brändas (Academic Press, 2007), Vol. 52, pp. 21-57.

${ }^{14}$ A. Muñoz, M. Fuss, M. A. Cortés-Giraldo, S. Incerti, V. Ivanchenko, A. Ivanchenko, J. M. Quesada, F. Salvat, C. Champion, and G. GómezTejedor, in Radiation Damage in Biomolecular Systems, edited by G. García Gómez-Tejedor and M. C. Fuss (Springer, Netherlands, 2012), pp. 203-225.

${ }^{15}$ R. D. White, W. Tattersall, G. Boyle, R. E. Robson, S. Dujko, Z. L. Petrovic, A. Bankovic, M. J. Brunger, J. P. Sullivan, S. J. Buckman, and G. Garcia, Appl. Radiat. Isot. 83(Pt. B), 77-85 (2014).

${ }^{16}$ J. de Urquijo, E. Basurto, A. M. Juarez, K. F. Ness, R. E. Robson, M. J. Brunger, and R. D. White, J. Chem. Phys. 141, 014308 (2014).

${ }^{17}$ R. D. White, M. J. Brunger, N. A. Garland, R. E. Robson, K. F. Ness, G. Garcia, J. de Urquijo, S. Dujko, and Z. L. Petrović, Eur. Phys. J. D 68, 1-6 (2014).

${ }^{18}$ S. Incerti, G. Baldacchino, M. Bernal, R. Capra, C. Champion, Z. Francis, P. Guèye, A. Mantero, B. Mascialino, P. Moretto, P. Nieminen, C.
Villagrasa, and C. Zacharatou, Int. J. Model. Simul. Sci. Comput. 1, $157-178$ (2010).

${ }^{19}$ J. Builth-Williams, S. M. Bellm, D. B. Jones, H. Chaluvadi, D. Madison, C. G. Ning, B. Lohmann, and M. J. Brunger, J. Chem. Phys. 136, 024304 (2012).

${ }^{20}$ D. B. Jones, S. M. Bellm, F. Blanco, M. Fuss, G. Garcia, P. Limão-Vieira, and M. J. Brunger, J. Chem. Phys. 137, 074304 (2012).

${ }^{21}$ D. B. Jones, S. M. Bellm, P. Limão-Vieira, and M. J. Brunger, Chem. Phys. Lett. 535, 30-34 (2012).

${ }^{22}$ Z. Masin, J. D. Gorfinkiel, D. B. Jones, S. M. Bellm, and M. J. Brunger, J. Chem. Phys. 136, 144310 (2012).

${ }^{23}$ M. Stener, P. Decleva, D. M. P. Holland, and D. A. Shaw, J. Phys. B: At. Mol. Phys. 44, 075203 (2011).

${ }^{24}$ F. Ferreira da Silva, D. Almeida, G. Martins, A. R. Milosavljevic, B. P. Marinkovic, S. V. Hoffmann, N. J. Mason, Y. Nunes, G. Garcia, and P. Limao-Vieira, Phys. Chem. Chem. Phys. 12, 6717-6731 (2010).

${ }^{25}$ P. Palihawadana, J. Sullivan, M. Brunger, C. Winstead, V. McKoy, G. Garcia, F. Blanco, and S. Buckman, Phys. Rev. A 84, 062702 (2011).

${ }^{26}$ M. C. Fuss, A. G. Sanz, F. Blanco, J. C. Oller, P. Limão-Vieira, M. J. Brunger, and G. García, Phys. Rev. A 88, 042702 (2013).

${ }^{27}$ A. Zecca, L. Chiari, G. Garcia, F. Blanco, E. Trainotti, and M. J. Brunger, J. Phys. B: At. Mol. Phys. 43, 215204 (2010).

${ }^{28}$ J. B. Maljkovic, A. R. Milosavljevic, F. Blanco, D. Sevic, G. Garcia, and B. P. Marinkovic, Phys. Rev. A 79, 052706 (2009).

${ }^{29}$ C. G. Ning, K. Liu, Z. H. Luo, S. F. Zhang, and J. K. Deng, Chem. Phys. Lett. 476, 157-162 (2009).

${ }^{30}$ S. H. R. Shojaei, B. Hajgató, and M. S. Deleuze, Chem. Phys. Lett. 498, 45-51 (2010).

${ }^{31}$ P. L. Levesque, M. Michaud, and L. Sanche, J. Chem. Phys. 122, 094701 (2005).

${ }^{32}$ M. H. Palmer, I. C. Walker, M. F. Guest, and A. Hopkirk, Chem. Phys. 147, 19-33 (1990).

${ }^{33}$ I. Nenner and G. J. Schulz, J. Chem. Phys. 62, 1747-1758 (1975).

${ }^{34}$ D. Almeida, D. Kinzel, F. Ferreira da Silva, B. Puschnigg, D. Gschliesser, P. Scheier, S. Denifl, G. García, L. González, and P. Limão-Vieira, Phys. Chem. Chem. Phys. 15, 11431-11440 (2013).

${ }^{35}$ D. Almeida, F. Ferreira da Silva, G. García, and P. Limão-Vieira, Phys. Rev. Lett. 110, 023201 (2013).

${ }^{36}$ F. Blanco, A. Muñoz, D. Almeida, F. Ferreira da Silva, P. Limão-Vieira, M. C. Fuss, A. G. Sanz, and G. García, Eur. Phys. J. D 67, 199 (2013).

${ }^{37}$ A. G. Sanz, M. C. Fuss, A. Muñoz, F. Blanco, P. Limão-Vieira, M. J. Brunger, S. J. Buckman, and G. García, Int. J. Radiat. Biol. 88, 71-76 (2012).

${ }^{38}$ A. Muñoz, J. M. Pérez, G. García, and F. Blanco, Nucl. Instrum. Methods Phys. Res., Sect. A 536, 176-188 (2005).

${ }^{39}$ A. G. Sanz, M. C. Fuss, F. Blanco, Z. Mašín, J. D. Gorfinkiel, F. Carelli, F. Sebastianelli, F. A. Gianturco, and G. García, Appl. Radiat. Isot. 83(Pt. B), 57-67 (2014).

${ }^{40}$ I. Linert, M. Dampc, B. Mielewska, and M. Zubek, Eur. Phys. J. D 66, 1-9 (2012)

${ }^{41}$ W. Y. Baek, A. Arndt, M. U. Bug, H. Rabus, and M. Wang, Phys. Rev. A 88, 032702 (2013).

${ }^{42}$ J. R. Ferraz, A. S. dos Santos, G. L. C. de Souza, A. I. Zanelato, T. R. M. Alves, M. T. Lee, L. M. Brescansin, R. R. Lucchese, and L. E. Machado, Phys. Rev. A 87, 032717 (2013)

${ }^{43}$ W. Wolff, H. Luna, L. Sigaud, A. C. Tavares, and E. C. Montenegro, J. Chem. Phys. 140, 064309 (2014).

${ }^{44}$ A. Muñoz, F. Blanco, G. García, P. A. Thorn, M. J. Brunger, J. P. Sullivan, and S. J. Buckman, Int. J. Mass Spectrom. 277, 175-179 (2008).

${ }^{45}$ CRC Handbook of Chemistry and Physics, edited by D. R. Lide (CRC Press, Boca Raton, FL, 2005), Internet Version 2005, http:// www.hbcpnetbase.com.

${ }^{46}$ R. Colmenares, A. G. Sanz, M. C. Fuss, F. Blanco, and G. García, Appl. Radiat. Isot. 83(Pt. B), 91-94 (2014).

${ }^{47} \mathrm{~T}$. Field, private communication (2008).

${ }^{48}$ M. C. Fuss, A. G. Sanz, F. Blanco, P. Limão-Vieira, M. J. Brunger, and G. García, Eur. Phys. J. D 68, 161 (2014).

${ }^{49}$ R. Mota, R. Parafita, A. Giuliani, M.-J. Hubin-Franskin, J. M. C. Lourenco, G. Garcia, S. V. Hoffmann, N. J. Mason, P. A. Ribeiro, M. Raposo, and P. Limão-Vieira, Chem. Phys. Lett. 416, 152-159 (2005). 